\section{Development of myasthenia gravis in a patient with chronic myeloid leukemia during treatment with nilotinib}

\author{
David Sanford, ${ }^{1}$ Maria MacDonald, ${ }^{2}$ \\ Michael Nicolle, 2,3 Anargyros Xenocostas ${ }^{1}$ \\ ${ }^{1}$ Department of Medicine, Division of \\ Hematology, 'Department of Oncology, \\ ${ }^{3}$ Department of Clinical Neurological \\ Sciences, University of Western Ontario, \\ London, ON, Canada
}

\begin{abstract}
We report on a patient diagnosed with chronic myeloid leukemia (CML) who developed myasthenia gravis while on treatment with nilotinib. Autoimmune disease, including the development of myasthenia gravis, has been described in association with CML as well as the use of tyrosine kinase inhibitors. Second generation tyrosine kinase inhibitors are highly effective in the treatment of CML, although can result in adverse effects related to off-target kinase inhibition, and longer term reporting of adverse effects is required.
\end{abstract}

\section{Case Report}

A 40-year-old man presented to our outpatient clinic with fatigue and night-sweats. He had splenomegaly on physical examination and his leukocyte count was elevated at $285 \times 10^{9} / \mathrm{L}$ with neutrophilia and marked left shift. Fluorescent in situ hybridization performed on peripheral blood leukocytes and bone marrow cells showed the $A B L 1 / B C R$ single fusion gene in $89 \%$ and $97 \%$ of cells, respectively. A bone marrow exam showed left shift with myeloid hyperplasia with $2 \%$ blasts. Cytogenetic analysis performed on the sample showed a karyotype of $46 \mathrm{XY} \mathrm{t}(9 ; 22 ; 12)$ (q34;q11.2;q13)[11]. The patient was started on the second-generation tyrosine kinase inhibitor (TKI) nilotinib $300 \mathrm{mg}$ orally twice daily. His constitutional symptoms improved and he initially reported no side effects from nilotinib. At 3 months, he attained a complete hematologic response and a 2.2 log reduction of his BCR-ABL fusion transcript levels by quantitative PCR testing.

Six months after starting nilotinib the patient presented to our clinic with a history of fluctuating diplopia and bilateral ptosis which were worse at the end of the day. He reported difficulty in performing push-ups secondary to new proximal arm weakness. Physical exam revealed diplopia in all directions of gaze. His diplopia and ptosis worsened after prolonged upward gaze and he had a positive ice pack test. He had mild fatigable weakness of head flexion and of the right deltoid. His neurologic exam was otherwise normal.

The patient was referred to a neurologist and a neuro-ophthalmologist for further assessment. Testing for acetylcholine receptor antibodies was positive. Repetitive nerve stimulation studies showed decremental response in the facial and spinal accessory nerves. Computed tomography of the chest ruled out thymoma. Magnetic resonance imaging of his brain and spinal cord was normal. The patient was referred to our local neuromuscular disorders clinic and was diagnosed with generalized seropositive myasthenia gravis. He was started on pyridostigmine as well as prednisone, which resolved his symptoms. He is currently considering a thymectomy. We elected to continue the patient on nilotinib as he was demonstrating a favorable response on this medication and was on target to achieve a major molecular response. We were also uncertain if the development of myasthenia gravis was triggered by nilotinib and whether his symptoms would improve with stopping the medication.

\section{Discussion}

There are many published reports of medication-induced myasthenia gravis or myasthenia-like symptoms. Medications implicated include: d-penicillamine, interferon- $\alpha$, ribavirin, fludarabine, statins, fluoroquinolones, carbamazepine and chloroquine. ${ }^{1-6}$

Tandutinib, a TKI that has been investigated for use in chronic myeloid leukemia (CML), acute myeloid leukemia and glioblastoma, has also been reported by Lehky and colleagues to cause a myasthenic-like syndrome. ${ }^{7}$ The authors of this study reported a series of 6 patients treated with tandutinib that developed clinical and electrophysiologic findings compatible with a myasthenic-like syndrome developing within 3-112 days of starting the drug. Testing for acetylcholine receptor antibodies was assessed in two of the six patients and these were negative in both. One patient had muscle specific kinase (MuSK) antibody testing, which was also negative. Symptoms spontaneously resolved in those that stopped tandutinib and repetitive nerve stimulation tests normalized in some patients. The authors of this study postulated that the findings might relate to activity of tandutinib against MuSK in the postsynaptic neuromuscular junction receptor.

To our knowledge, there have been no other reports of myasthenia gravis developing in
Correspondence: David Sanford, Department of Medicine, Division of Hematology, London Health Sciences Centre, Room E6-208, Victoria Hospital, 800 Commissioners Road East, London N6A 4W9, ON, Canada.

Tel.: $+1.519 .685 .8500 / 55232$.

Fax: +1.519.685.8477.

E-mail: dsanfor@uwo.ca

Key words: chronic myeloid leukemia, tyrosine kinase inhibitors, adverse drug events.

Contributions: DS and AX wrote prepared the manuscript; MM and MN provided clinical information around the case and helped in editing the manuscript.

Conflict of interests: the authors declare no potential conflict of interests.

Received for publication: 7 January 2014. Accepted for publication: 24 March 2014.

This work is licensed under a Creative Commons Attribution NonCommercial 3.0 License (CC BYNC 3.0).

(C) Copyright D. Sanford et al., 2014

Licensee PAGEPress, Italy

Hematology Reports 2014; 6:5288

doi:10.4081/hr.2014.5288

patients treated with nilotinib or imatinib and dasatinib. In contrast to the previous report of tandutinib causing myasthenia gravis, our patient had positive acetylcholine receptor antibodies, confirming a diagnosis of immune myasthenia gravis. This suggests that if nilotinib caused myasthenia gravis in our patient, it may involve a different mechanism than tandutinib. There have been reports of other TKIs inducing autoimmune disease. Rea and colleagues described a lupus-like syndrome developing in a 76 year-old patient with CML using dasatinib. ${ }^{8}$ Their patient developed fever, arthralgia, hepatosplenomegaly, pleurisy and strongly positive serologic tests for ANA and double-stranded DNA. There was clinical and serologic normalization after stopping the drug.

It is also possible that the development of myasthenia gravis in our patient could have resulted from his underlying CML and disturbed immune regulation related to this. The association between myasthenia gravis and thymomas and Lambert-Eaton syndrome with extra-thymic malignancies, particularly SCLC and perhaps lymphoproliferative malignancies is well known and might related to immune dysregulation. ${ }^{9}$ The discovery of an underlying solid-tumor appears to be more frequently reported than hematologic malignancy. ${ }^{9}$ There are however several published case reports describing an association between the development of myasthenia gravis both preceding 
and following the development of CML. ${ }^{10-15}$ The causal association between these two conditions cannot be determined from these individual reports.

\section{Conclusions}

It is uncertain if the development of myasthenia gravis in our patient was related to use of nilotinib, his underlying CML or neither. Given the relatively rare incidence rate of both CML and myasthenia, it may be possible that myasthenia may be an uncommon but serious side effect of nilotinib. Most of the TKIs currently approved for use in CML, have been reported to have some immunomodulatory effects, at least in vitro. Longer-term post-marketing research on nilotinib and other TKIs may help to clarify if these medications are associated with increased risk for developing myasthenia gravis and other autoimmune disease.

\section{References}

1. Rossi M, Lusini G, Biasella A et al. Pruli- floxacin as a trigger of myasthenia gravis. $\mathrm{J}$ Neurol Sci 2009;280:109-10.

2. Dionisiotis J, Zoukos Y, Thomaides T. Development of myasthenia gravis in two patients with multiple sclerosis following interferon beta treatment. J Neurol Neurosurg Psychiatry 2004;75:1079.

3. Rasmussen, M. Carbamazepine and myasthenia gravis. Neuropediatrics 2004;35:259.

4. Robberecht W, Bednarik J, Bourgeois P et al. Myasthenic syndrome caused by direct effect of chloroquine on neuromuscular junction. Arch Neurol 1989;46:464-8.

5. Fujimaki K, Takasaki H, Koharazawa H, et al. Idiopathic thrombocytopenic purpura and myasthenia gravis after fludarabine treatment for chronic lymphocytic leukemia. Leuk Lymphoma 2005;46:1101-2.

6. de Sousa, E, Howard, J. More evidence for the association between statins and myasthenia gravis. Muscle Nerve 2008;38:1085-6.

7. Lehky TJ, Iwamoto FM, Kreisl TN, et al. Neuromuscular junction toxicity with tandutinib induces a myasthenic-like syndrome. Neurology 2011;76:236-41.

8. Rea D, Bergeron A, Fieschi C, et al. Dasatinib-induced lupus. Lancet 2008;372: 713-4.

9. Levin N, Abramsky 0, Lossos A, et al.
Extrathymic malignancies in patients with myasthenia gravis. J Neurol Sci 2005;237: 39-43.

10. Pavithran K, Panakkel C, Roy D, et al. Chronic myeloid leukaemia in a man with myasthenia gravis treated by thymectomy. Eur J Haematol 2002;69:105-7.

11. Aguiar RC, Beitler B, Dorlhiac-Llacer PE, et al. Secondary chronic myelogenous leukemia: a diverse pathogenesis? Acta Haematol 1994;92:101-3.

12. Wanders J, Wattendorff AR, Endtz LJ, et al. Chronic myeloid leukemia in myasthenia gravis after long-term treatment with 6mercaptopurine. Acta Med Scand 1981; 210:235-8.

13. Kumar P, Jain P, Menon H, Purvish P. Chronic myeloid leukemia presenting as paraneoplastic ocular myasthenia gravis. Ann Oncol 2007;18:804-5.

14. Pérez A, Perella M, Pastor E, et al. Myasthenia gravis induced by alpha-interferon therapy. Am J Hematol 1995;49:365-6.

15. Djaldetti M, Pinkhas J, de Vries A, et al. Myasthenia gravis in a patient with chronic myeloid leukemia treated by busulfan. Blood 1968;32:336-40. 